\title{
Flowpath 2017 - National Meeting on Hydrogeology, Cagliari, 14-16 June 2017
}

\section{Flowpath 2017 - Conferenza Nazionale sull'Idogeologia, Cagliari, 14-16 giugno 2017}

Giorgio Ghiglieri Chair della Conferenza e Comitato esecutivo - Università degli Studi di Cagliari - ghiglieri@unica.it -

Stefania Da Pelo Chair della Conferenza e Comitato esecutivo - Università degli Studi di Cagliari - sdapelo@unica.it

Gabriele Uras Comitato esecutivo - Università degli Studi di Cagliari - urasg@unica.it

Keywords: hydrogeology, IAH Italian Chapter, meeting.

Parole chiave: idrogeologia, Comitato IAH, conferenza.

A tre anni dalla seconda edizione, tenutasi a Viterbo nel 2014, il terzo Meeting Nazionale della Sezione Italiana dell'International Association of Hydrogeologist (IAH), Flowpath 2017, è stato organizzato tra il 14 e il 16 giugno dal Dipartimento di Scienze Chimiche e Geologiche dell'Università di Cagliari, con la collaborazione del Comitato Italiano dell'IAH (IAH Italian Chapter), presso la sede di Cagliari della Fondazione di Sardegna. Lorganizzazione ha potuto beneficiare del contributo di numerosi sponsor e patrocini e soprattutto del supporto economico dell'Università degli studi di Cagliari e del Dipartimento di Scienze Chimiche e Geologiche, che hanno appoggiato l'iniziativa già dalle prime fasi organizzative (http://ojs.unica.it/index.php/flowpath2017/pages/view/sponsorship).

Erano presenti alla cerimonia di apertura del Convegno la professoressa Alessandra Carucci, delegato del Rettore e Prorettore all'internazionalizzazione dell'Università di Cagliari, la dottoressa Gabriella Mulas (direttore del Servizio tutela e gestione delle risorse idriche, vigilanza sui servizi idrici e gestione delle siccità - ARDIS - Regione Autonoma della Sardegna), il dottor Davide Boneddu (presidente dell'Ordine dei geologi della Sardegna), i professori Giulio Barbieri, Giovanni Barrocu, Antonio Pala e Antonio Vernier, quali membri del comitato d'onore (Fig. 1).

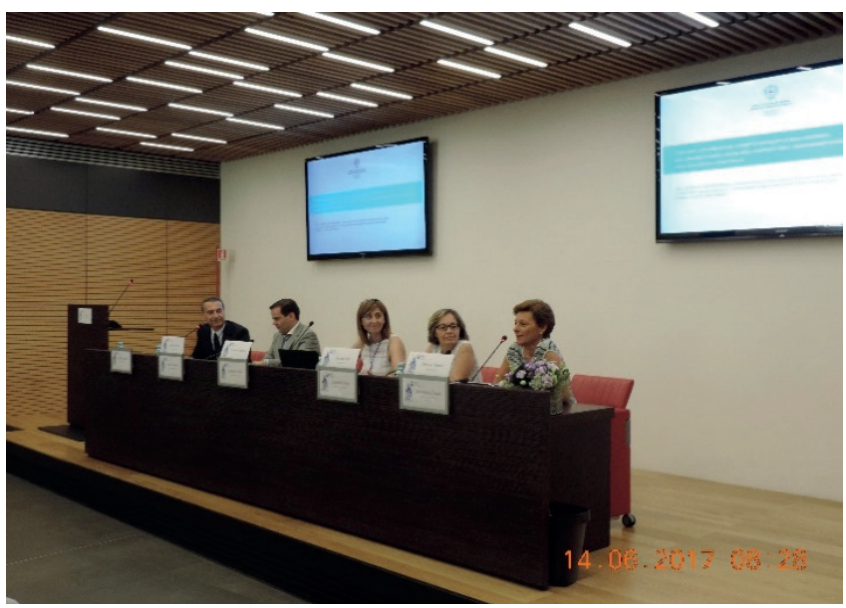

Il congresso si è articolato in quattro sessioni, con presentazioni orali e poster, ed eventi sociali. Le quattro sessioni hanno trattato i seguenti temi: la qualità delle acque sotterranee $e$ le misure per la loro protezione dall'inquinamento, l'idrogeologia degli acquiferi fessurati e carsici, i modelli di flusso e trasporto nelle acque sotterranee e la gestione delle risorse idriche sotterranee nelle regioni aride e semiaride. Sono stati presentati 77 contributi scientifici da studiosi italiani e stranieri provenienti da 15 nazioni dell'Europa, degli USA e dell'Africa. Tutti i contributi sono stati sottoposti a una procedura di peer review, grazie alla preziosa collaborazione dei membri del Comitato Scientifico.

Al convegno erano presenti circa 120 partecipanti, tra ricercatori ed esponenti del mondo professionale, provenienti da tutto il territorio nazionale e anche dall'estero. L'appuntamento scientifico - i cui atti sono pubblicati sulla piattaforma open access dell'Ateneo di Cagliari (http://ojs.unica.it/index. php/flowpath2017/index) - costituisce un'opportunità per il mondo scientifico e professionale in campo idrogeologico per scambiare idee ed esperienze sulle diverse problematiche riguardanti le acque sotterranee. Privilegiando la partecipazione dei giovani idrogeologi e volendo incoraggiare a contribuire al miglioramento della gestione delle risorse idriche, obiettivo della conferenza è stato quello di promuovere il dia-

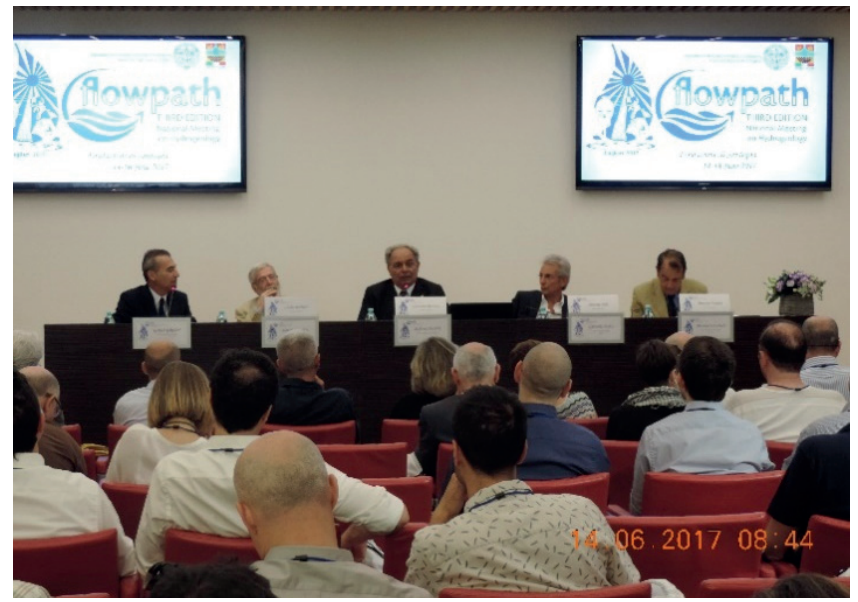

Fig. 1: Cerimonia di Apertura del Flowpath 2017

Fig. 1: Opening ceremony of Flowpath 2017. 
logo e lo scambio di conoscenza, di approfondire gli aspetti teorici e pratici della circolazione idrica sotterranea, di aggiornare i portatori di interesse, i ricercatori e i professionisti sugli sviluppi tecnici e scientifici raggiunti in campo idrogeologico. Riassunti estesi di un certo numero di lavori saranno pubblicati sui Rendiconti online della Società Geologica Italiana, rivista indicizzata dalle principali banche dati internazionali quali Scopus e WoS. Una selezione sarà pubblicata su Acque sotterranee - Italian Journal of Groundwater.

All'inizio di ogni sessione tematica vi è stata la presentazione di una relazione ad invito tenuta da esperti di fama internazionale. Il primo contributo ha trattato il tema dell'individuazione dei valori di fondo nei sistemi acquatici (Prof.ssa Rosa Cidu), il secondo ha analizzato i fattori che controllano l'origine e le proprietà dei sistemi di frattura negli acquiferi fessurati (Dr Robert Wyns), il terzo ha presentato gli strumenti isotopici utilizzabili nella valutazione dell'efficienza dei sistemi che inducono i processi di denitrificazione nelle acque sotterranee (Prof. Albert Soler Gil) e l'ultimo le sfide e le opportunità che si presentano nella gestione delle acque sotterranee nelle regioni aride e semi aride (Dr Viviana Re). Durante le sessioni sono state presentate un totale di 34 presentazioni orali e sono stati esposti 43 poster. Nella scelta dei relatori sono stati privilegiati i lavori presentati da giovani, che hanno esposto circa l' $85 \%$ delle presentazioni orali (http://convegni.unica.it/ flowpath2017/files/2017/06/FINAL-PROGRAMME_.pdf). I principali temi trattati durante la prima sessione hanno riguardato la vulnerabilità degli acquiferi, la contaminazione da nitrati, l'approccio isotopico per la valutazione dell'origine degli inquinanti e per il raffinamento dei modelli concettuali idrogeologici, l'utilizzo della temperatura quale tracciante naturale per caratterizzare le interazioni fra acque superficiali e sotterranee, gli strumenti statistici per la valutazione dei valori di fondo naturali, la gestione delle acque sotterranee in aree urbane, il monitoraggio delle aree contaminate o soggette all'intrusioni saline e la valutazione dell'impatto sulle acque sotterranee dei sistemi geotermici a bassa entalpia, il cui utilizzo è in continua crescita negli ultimi anni. Nella seconda sessione, orientata alla caratterizzazione degli acquiferi fessurati, le presentazioni si sono focalizzate soprattutto sulla valutazione della ricarica in acquiferi carsici, sugli strumenti e le metodologie di valutazione dei parametri idrodinamici degli acquiferi fessurati e sugli effetti dei recenti sismi sulla circolazione delle acque sotterranee. Durante la terza sessione, dedicata agli strumenti modellistici, è stato posto l'interesse sull'utilizzo della ricostruzione dei modelli geologici 3D per fissare le condizioni di limite e al contorno dei modelli numerici di flusso delle acque sotterranee, sui modelli di trasporto reattivo dei contaminanti nello spazio $1 \mathrm{D}, 2 \mathrm{D}$ e $3 \mathrm{D}$ e sulle varie opportunità di utilizzo di piattaforme open source per la modellistica idrogeologica e la gestione delle acque sotterranee. Ultima, ma non di importanza, la quarta sessione si è concentrata sulla gestione delle acque sotterranee in aree aride e semiaride, in aree carsiche e in aree interessate da elevati tenori naturali di contaminanti. E' stato un esempio il lavoro, condotto nell'ambito di un progetto H2020 (FLOWERED), presentato dai ricercatori dell'Università di Cagliari sulla ricostruzione delle condizioni geologiche e idrogeologiche che condizionano i processi di arricchimento da fluoro nelle acque sotterranee del Rift dell'Africa orientale. Ulteriori temi di interesse hanno riguardato le tecniche di ricarica artificiale degli acquiferi e le metodologie indirette di ricostruzione del bilancio idrologico per la valutazione della ricarica naturale in bacini non strumentati. La quarta sessione è stata dedicata al Prof. G.M. Zuppi, scomparso nel 2011. Da allora, durante le edizioni del Flowpath, viene consegnato il Premio omonimo per la migliore tesi di Dottorato in campo idrogeologico. La terza edizione è stata assegnata alla Dott.ssa Mariachiara Caschetto che ha avuto anche la possibilità di presentare il suo lavoro durante la quarta sessione del Convegno. La cerimonia di consegna del premio, tenutasi durante la cena sociale, è stata resa ancora più piacevole dalla suggestiva cornice del faro di capo S. Elia e del mare di Calamosca (Fig. 2). Gli oltre 100 partecipanti alla cena hanno potuto apprezzare il piacere del dialogo scientifico davanti a piatti e bevande tipiche della Sardegna.

Al termine della prima giornata di lavori si è tenuta l'annuale riunione dei soci italiani IAH, presieduta dalla Presidente Prof.ssa Daniela Ducci. I soci IAH italiani hanno raggiunto i 184 iscritti, superiori a Germania e Francia e terzi dietro Inghilterra e Spagna. Durante l'assemblea è stato annunciato il prossimo Flowpath 2019 che si terrà a Milano con un'organizzazione congiunta delle tre sedi del Politecnico di Milano, dell'Università degli studi di Milano e dell'Università di Milano-Bicocca. Nel 2020 si terrà, probabilmente a Caserta, il " 3 rd International Multidisciplinary Conference on Mineral Water - MinWat 2020. L'evento sarà sponsorizzato da Ferrarelle SpA.

Ulteriore interessante contributo ha riguardato il report del Prof. Petitta sulla "Produzione e produttività della Comunità Idrogeologica Italiana".

Al termine della seconda giornata si è tenuto un interessante evento organizzato dai giovani idrogeologi dell'Early Career Hydrogeologists' Network (ECHN) sulle sfide e le opportunità dell'idrogeologia urbana. Nonostante l'evento fosse dedicato principalmente ai giovani, anche gli esperti hanno partecipato numerosi al coinvolgente intervento del Prof. Marco Masetti.

La terza giornata è stata interamente dedicata al Field Trip nell'area del Sulcis Iglesiente. Dopo un piccolo intoppo dovuto a un'avaria al motore di uno degli autobus, risolto in maniera egregia dal lavoro di squadra di tutto lo staff di organizzazione, la giornata si è svolta secondo i programmi. Le prime tappe del Field trip hanno compreso la visita all'opera mineraria di Porto Flavia e alla discordanza ordoviciana osservabile lungo la passeggiata panoramica di Nebida. Sullo sfondo il suggestivo paesaggio dello scoglio di Pan di Zucchero e della Laveria Lamarmora Lassetto idrogeologico dell'anello metallifero (formazione carbonatico-dolomitica del Cambriano ospitante le più importanti mineralizzazioni di $\mathrm{Zn}$ e $\mathrm{Pb}$ di Italia) ha condizionato l'attività di questa importante provincia mineraria della Sardegna, spingendo a sempre più avanzate 
tecniche per l'abbattimento della piezometrica all'interno dei cantieri di coltivazione. Un gustoso pranzo a base di tonno e altri piatti tipici della zona è stato consumato presso la Tonnara di Portoscuso, gentilmente messa a disposizione dall'amministrazione comunale. In quest'area insiste uno dei grandi poli industriale perimetrati come Siti di Interesse Nazionale per la bonifica: Portovesme. La giornata si è conclusa con un breve documentario proiettato dalla Ligestra Srl sulle attività di messa in sicurezza della falda delle aree ex Alumix. I

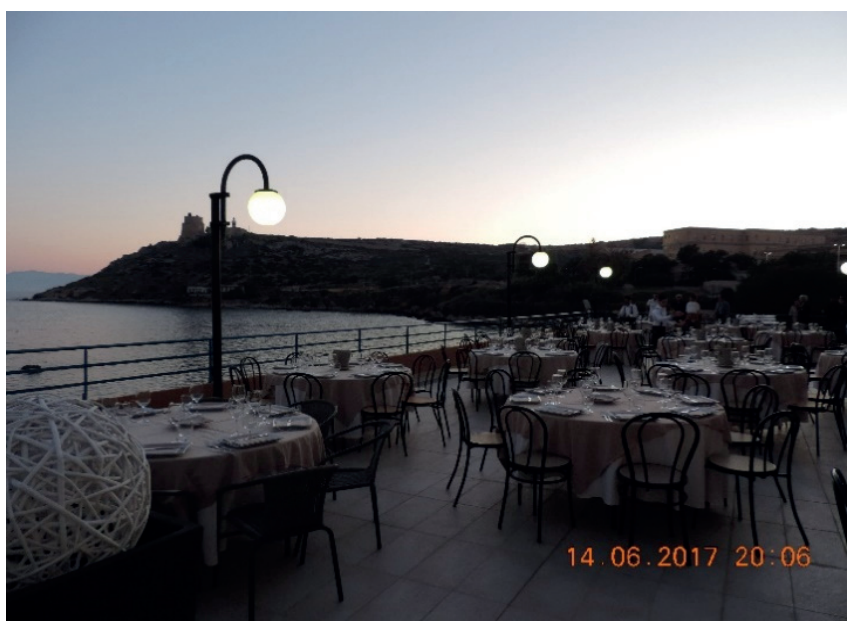

partecipanti al Convegno hanno mostrato molto interesse per l'iniziativa scientifica, oltre che per la cornice nella quale si sono svolte tutte le attività: dalla sede della Fondazione alla sala messa a disposizione per i coffee break e i pranzi dal Convitto Nazionale Vittorio Emanuele e a tutte le bellezze della città di Cagliari; dalle aree minerarie al sito di Portoscuso. Quest'ultimo, al di là della fama di sito industriale contaminato, custodisce delle bellezze naturali dell'ambiente costiero e urbano uniche e meritevoli di essere visitate.

Fig. 2: Location della cena sociale e cerimonia di consegna del Premio Zuppi.

Fig. 2: Gala Dinner location and and the 3rd Zuppi award ceremony.
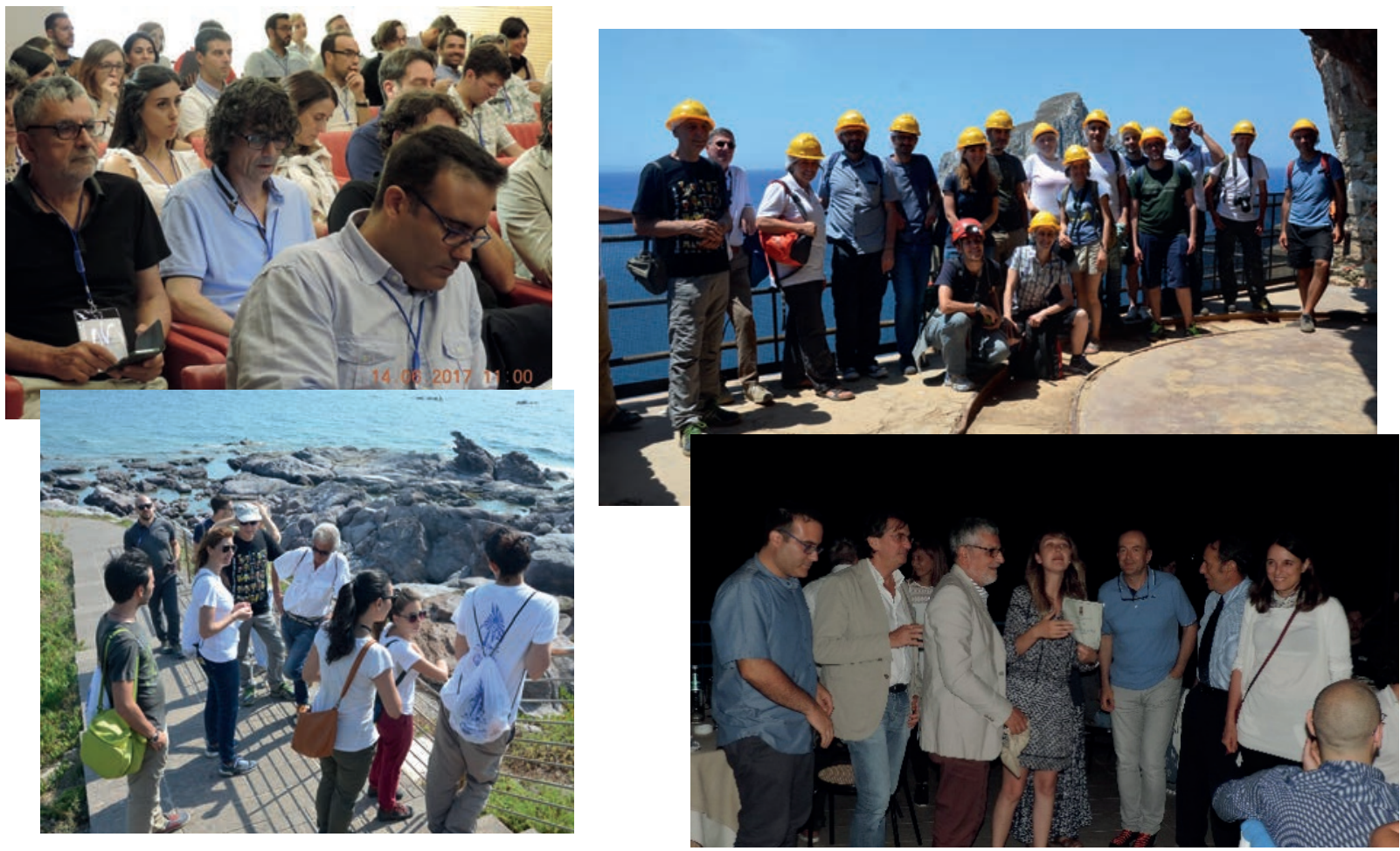

Vari momenti del convegno.

Shots during the Flowpath 2017 Conference. 